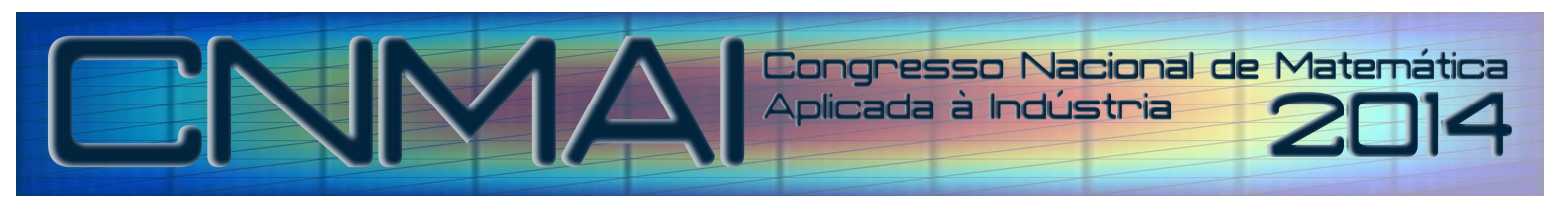

18 a 21 de novembro de 2014, Caldas Novas - Goiás

\title{
USO DE SISTEMAS AUTOMATIZADOS PARA OTIMIZAR A CAPTAÇÃO DE ENERGIA EM PAINEIS SOLARES
}

\author{
Medeiros, I. P. M., isaacmedeiros.rn@gmail.com ${ }^{1}$ \\ Fontes, F. A. O., franciscofontes@uol.com.br ${ }^{1}$ \\ Lima, C. M., geracao3d@gmail.com ${ }^{1}$ \\ Barbosa, C. R. F., cleiton@dem.ufrn.br ${ }^{1}$ \\ Valcacer, S. M., samara.valcacer@gmail.com² \\ Sanabio, R. G., robson.sanabio@uece.br ${ }^{2}$ \\ Dantas, V. B., valter.fisic@hotmail.com²
}

\begin{abstract}
${ }^{1}$ Universidade Federal do Rio Grande do Norte - UFRN 1 - Programa Pós-Graduação em Engenharia Mecânica PPgEM, Caixa Postal 1524 - Campus Universitário Lagoa Nova, CEP 59078-970, Natal/RN - Brasil 1

${ }^{2}$ Universidade Federal do Rio Grande do Norte - UFRN 2 - Programa Pós-Graduação em Ciência e Engenharia de Materiais - PPgCEM, Caixa Postal 1524 - Campus Universitário Lagoa Nova, CEP 59078-970, Natal/RN - Brasil 2
\end{abstract}

Resumo: As fontes de energia podem ser convencionais ou alternativas. Apesar de a energia convencional ser caracterizada pelo baixo custo, a energia alternativa ganha visibilidade e entre elas a solar, por ser limpa, silenciosa e poder adaptar-se aos mais diversos locais. A grande problemática no uso dessa energia é sua baixa eficiência na conversão de energia elétrica que é produzida a partir dos raios solares. E para ter sua eficiência energética melhorada o trabalho propõe um mecanismo de rastreamento acoplado ao painel solar para orientação dos movimentos a partir da incidência dos raios solares na superfície da célula solar Para proporcionar essa movimentação usou-se dois servomotores e um sistema embarcado com sensores de luz (LDR - 5mm) diretamente ligados aos servo mecanismos. O software embarcado utiliza linguagem $C$ que permite a determinação da posição correta do painel solar e com o uso desses mecanismos espera-se coletar a radiação solar máxima, aumentando assim a eficiência do conjunto utilizando-se um aparato de baixo custo.

Palavras-chave: Automação, Energia Solar, Microcontrolador, Linguagem C

\section{INTRODUÇÃO}

No mundo em que vivemos há uma grande demanda por energia elétrica e consequentemente energias renováveis. As fontes de energia renovável ganham espaço, pois, a energia proveniente dos combustíveis fósseis acabará em algum momento, além de ser uma fonte energética poluente.

Uma das formas mais comuns de conversão direta dessa energia em eletricidade é através de células fotovoltaicas. No entanto, uma célula estática possui limitações na captação de energia solar devido a sua imobilidade. Com isso em mente, pensou-se que se a célula se movesse em direção à fonte de energia luminosa, ela poderia gerar mais energia. Assim os painéis solares devem ser montados em um ângulo de 10 a 15 graus mais latitude do local e se move em direção ao sol.

Sendo a maioria das aplicações em painéis fixos, na medida em que os raios solares mudam o ângulo de incidência, com o passar do dia, ou mesmo com a mudança das estações do ano, têm-se uma perda significativa na captação dos raios solares.

O principal objetivo da pesquisa e conseguir que a célula fotovoltaica mova-se, direcionando-se à fonte de maior energia luminosa e converta energia elétrica. Bem como estudar a viabilidade econômica do dispositivo. Optando-se por usar um sistema de automação de baixo custo. 


\section{FUNDAMENTAÇÃO TEORIA}

\subsection{Radiação Solar}

O aproveitamento mais utilizado para conversão da energia solar em elétrica é a partir das células fotovoltaicas. Isso por ser uma energia infinita, limpa e silenciosa. Uma problemática quanto a seu uso é a baixa eficiência na conversão da energia, no máximo $30 \%$ dos raios solares são transformados em energia elétrica.

Uma forma de melhorar o aproveitamento da radiação solar deve ser ajustando à posição do painel solar de acordo com a latitude local e o período do ano em que se requer mais energia. No Hemisfério Sul, por exemplo, um sistema de captação solar fixo deve ser orientado para o Norte, com ângulo de inclinação similar ao da latitude local.

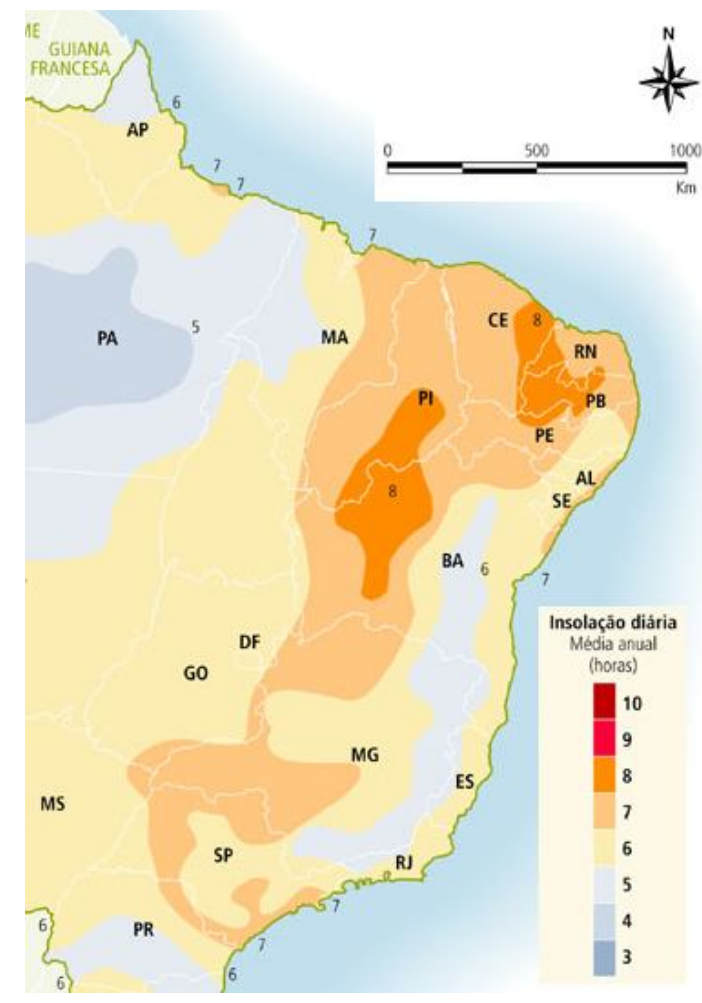

Figura 1: Mapa Solarimétrico do Brasil, parte região Nordeste Fonte: CRESESB.

Já na Região Nordeste que apresenta uma cor laranja (Figura 1) tem uma insolação média anual alta, sendo assim, viável a geração de energia solar. A radiação solar atinge a superfície terrestre, devido à reflexão e absorção dos raios solares pela atmosfera. Estima-se que a energia solar incidente sobre a superfície terrestre seja da ordem de 10 mil vezes o consumo energético mundial (CRESESB, 1999).

A Atlas Solarimétrico do Brasil (2000) apresenta uma estimativa da radiação solar incidente no país, resultante da interpolação e extrapolação de dados obtidos em estações solarimétricas distribuídas em vários pontos do território nacional. Devido, porém, ao número relativamente reduzido de estações experimentais e às variações climáticas locais e regionais, a Atlas de Irradiação Solar no Brasil faz estimativas da radiação solar a partir de imagens de satélites. Como pode ser visto, os maiores índices de radiação são observados na região Nordeste, com destaque para o Vale do São Francisco (CRESESB, 1999).

\subsection{Movimento de rastreamento solar}

O dispositivo utilizado para orientação do painel, concentra a energia de forma que o painel esteja sempre voltado para o raio solar, para que haja uma maior incidência de raios perpendiculares á superfície dos mesmo. A eficiência do sistema aumenta com a maior incidência dos raios solares.

São considerados paramentos para o deslocamento: declividade terrestre, latitude, ângulo horário, dia do ano e etc. $\mathrm{Na}$ maioria dos aplicativos de sistema ativos de seguimento, os sensores da placa de sombreamento são suficiente para determinar a posição solares através sistema eletrônico. (Alcântara, 2008). 


\subsection{Movimento em dois eixos para o suporte de painéis fotovoltaicos}

O movimento em dois eixos rastreador solar é um dispositivo utilizado para orientar um arranjo de painéis fotovoltaicos, concentradores de energia, de forma que estejam sempre voltados para o Sol, para que haja uma maior incidência de raios solares perpendiculares à superfície dos mesmos. Quando têm apenas um eixo, este pode ter orientação polar, norte-sul ou leste oeste. Com dois eixos, um deles é vertical para ajustar o azimute da superfície e o outro é horizontal, para ajustar a inclinação dos painéis (Paiva, 2009).

O rastreador em dois eixos tem movimentação azimutal (eixo vertical) e de inclinação (eixo horizontal) o que proporciona um grande rendimento de conversão da componente direta da radiação solar. Um aumento na concentração da irradiação pode ser conseguido com o auxilio de espelhos ou lentes. Os ângulos e a movimentação dos eixos deste sistema são mostrados na Figura 2.

Os ângulos deste modelo são os mesmos. A Equação (1) do ângulo de incidência direta é bastante simplificada devido ao movimento dos eixos horizontal e vertical, fazendo com que o ângulo de incidência direta seja igual à zero. $\mathrm{O}$ movimento em torno eixo vertical faz a correção do azimute, com isso o azimute do coletor solar ficará igual ao azimute do sol.

A intensidade da radiação solar exprime-se em geral pelo fluxo de energia, a energia por unidade de tempo (IN) incidente sobre uma superfície plana perpendicular aos raios solares. As unidades no sistema SI são W/m². Se a incidência não for normal, define-se o ângulo de incidência i como sendo o ângulo entre os raios solares e a normal à superfície (Falcão, Cornwall, 2014).

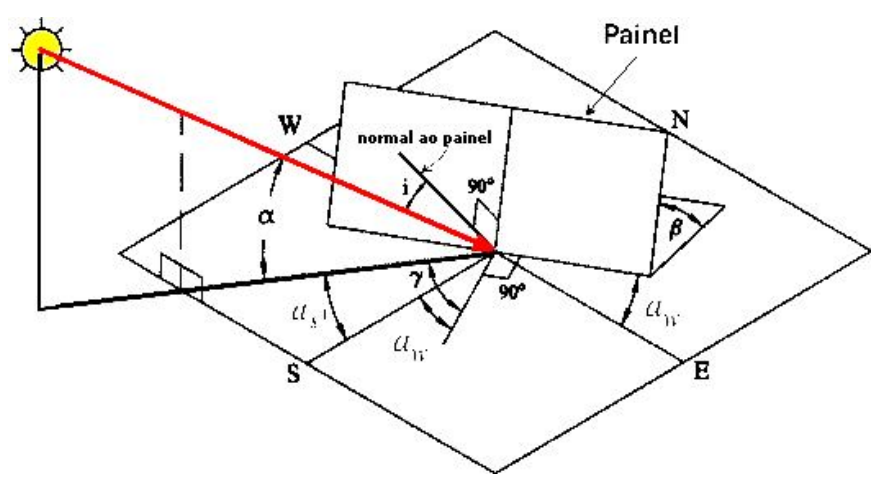

Figura 2: Incidência da radiação solar, Cornwall (2014).

$$
\operatorname{com} \alpha \mathrm{s}>0, \alpha \mathrm{w}<0
$$

No caso de incidência não normal $(/ \neq 0)$, o fluxo de energia recebido pela superfície por unidade de área, é Ic = IN $\cos /$

O ângulo de incidência / pode ser relacionado com o ângulo de altitude solar $\alpha$, com o ângulo de azimute solar $\alpha$, e com os dois ângulos que definem a orientação do painel: o ângulo $\beta$ de inclinação do painel (em relação ao plano horizontal) e o ângulo de azimute do painel $\alpha \mathrm{w}$ com Eq. (2) (formado pela direção Sul-Norte com plano horizontal da normal Eq. (3) ao painel; à convenção se sinal para $\alpha \mathrm{w}$ é a mesma que para $\alpha$ s). Essa relação é

$$
\cos /=\cos \alpha \cos (\alpha \mathrm{s}-\alpha \mathrm{w}) \sin \beta+\sin \alpha \cos \beta
$$

No caso de incidência normal,

$$
\alpha \mathrm{s}=\alpha \mathrm{w}, \alpha=90^{\circ} \mathrm{e} \mid=0 \text {. Se o painel for horizontal, fica } \beta=0 \text { e } /=90^{\circ} .
$$

A radiação solar é sempre maior numa área que se estende perpendicularmente em relação aos raios solares, do que numa área horizontal das mesmas dimensões. Uma vez que o azimute e a altura solar mudam ao longo do dia e do ano, o ângulo de incidência da radiação solar varia constantemente na maior parte das áreas potenciais ao aproveitamento da energia solar. A análise da radiação anual ajuda a equacionar a conveniência das áreas existentes, tendo em conta o seu aproveitamento solar.

A orientação da instalação solar tem por resultado diferentes níveis de irradiação. Um ângulo de $35^{\circ}$ de inclinação, no Brasil, o painel deve estar voltado para o Norte geográfico com uma inclinação de $25^{\circ}$ a $30^{\circ}$. Neste caso, o nível de irradiação é quinze por cento, maior do que numa área horizontal (ângulo de inclinação: $\beta=0$ ) (Falcão, Cornwall, 2014). 


\section{METODOLOGIA}

O projeto foi dividido em duas partes: a primeira foi o desenvolvimento da parte eletrônica e a segunda do algoritmo. O diagrama 1 apresenta um resumo do projeto.

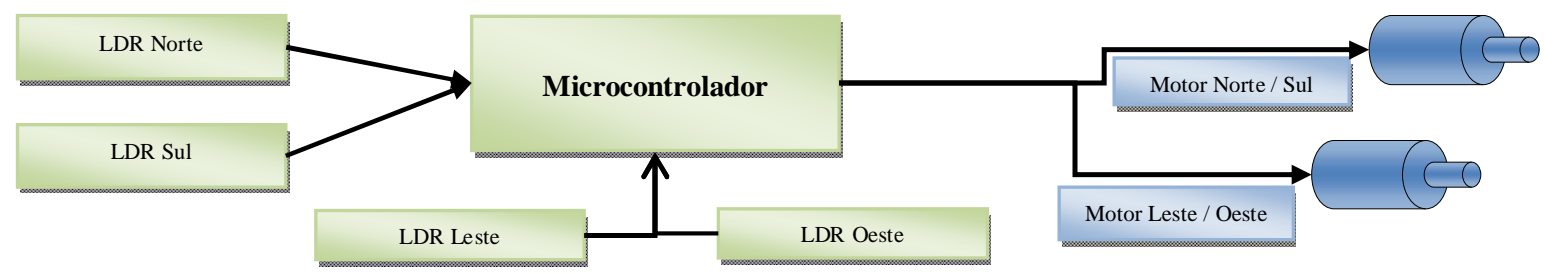

Diagrama 1: Diagrama representativo do sistema embarcado. Fonte: Autor

A proposta de implementação com a montagem de e também de prática aplicação para futuros projetos, foram pesquisados produtos de fácil acesso no mercado. Para esta fase inicial, foram utilizados 4 (quatro) sensores (5mm) LDR, 4 (quatro) resistores de $460 \Omega(\mathrm{Ohm}),+/-5 \%$, são cabos com ponteiras específicas para o uso microcontrolador (Arduino UNO) e dois servomotores 9G (modelo ser-gls09).

São 2 (Dois) servemotores, um para cada eixo cardeal. O movimento do painel em torno do eixo vertical é proporcional à diferença entre a irradiância útil recebida pelos 4 (quatro) sensores da placa de sombreamento Norte/Sul e Leste/Oeste. As saídas são as variáveis utilizadas pelo microcontrolador para a realização do controle dos movimentos em torno dos eixos vertical (azimute) e horizontal (elevação).

A Figura 3 ilustra o sistema eletro-mecânico utilizado que proporciona o encontro do sol perpendicularmente e igualitáriamente a superfície de cada um dos sensores. Os materiais utilizados são: (A) uma chapa de alumínio quadrada medindo $150,5 \mathrm{~mm}$ de cada lado e espessura de $2 \mathrm{~mm}$, uma caixa de luz $4 \times 4$ e uma chapa de alumínio quadrada com dimensões de $14,4 \mathrm{~mm}$ de cada lado, para o suporte do painel. (B) um perfil tubular do tipo quadrado de alumínio com dimensão $260 \mathrm{~mm}$ e/ou 1.1/32 polegada e $2 \mathrm{~mm}$ espessura, em (C) um servemotor que pesa $9 g$ foi colocado dentro do tubo para proporcionar o movimento em direção ao sol. (D) um perfil tubular do tipo redondo de alumínio com dimensão de $3 \mathrm{~mm}$ e/ou 1/8 polegadas foi utilizado para montar o suporte vertical garantindo assim a estabilidade do sistema.

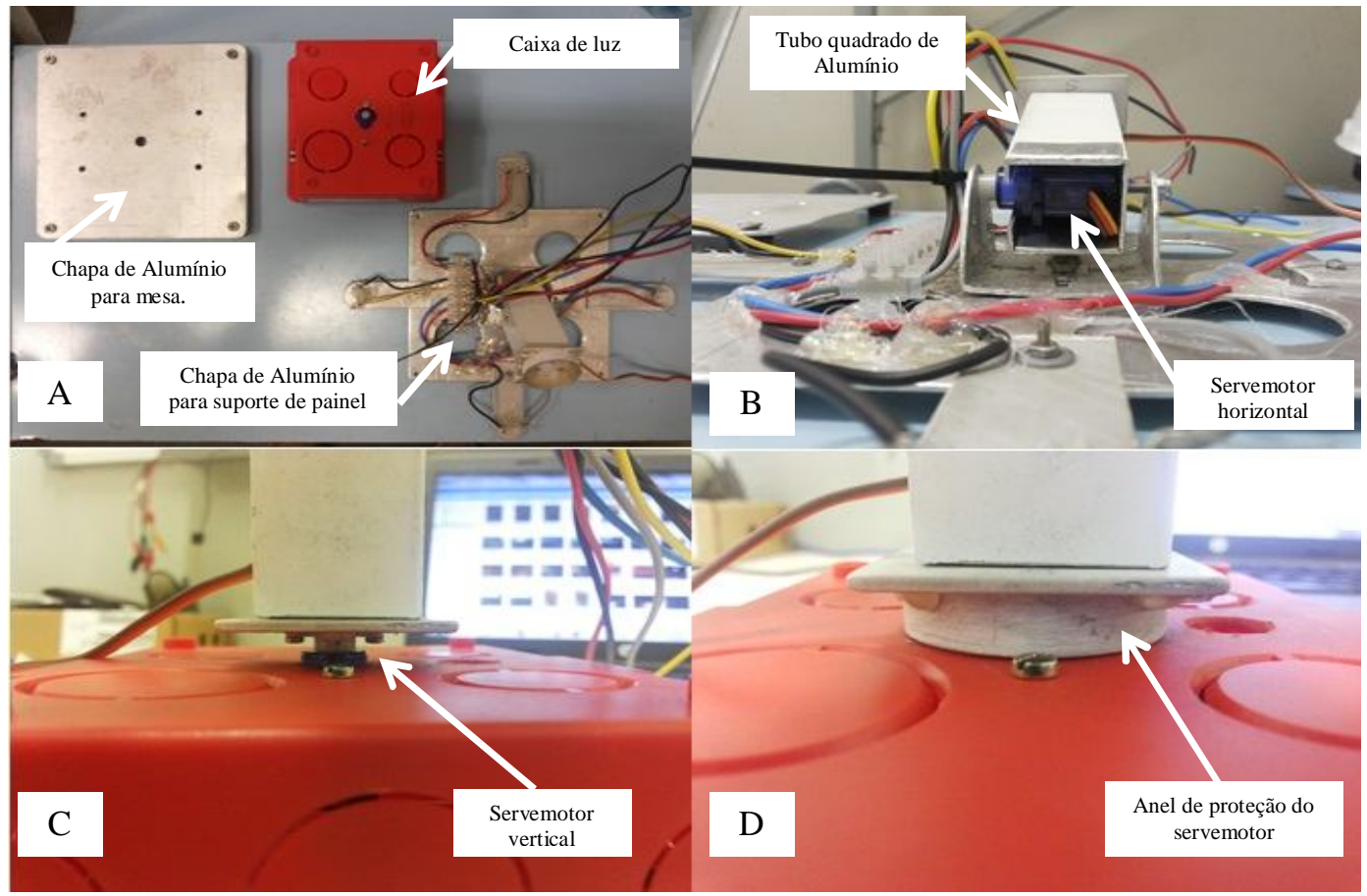

Figura 3: Visão geral, implementação de um painel fotovoltaico. Fonte: Autor.

Geralmente o dispositivo interfere no funcionamento do controlar para que o componente se movimente junto, e assim o ângulo de incidência dos raios solares sobre a superfície do painel seja o mesmo sobre a superfície do sensor. 


\subsection{Microcontrolador}

O microcontrolador Arduino parte do conceito de hardware e software livre e está aberto para uso e contribuição de toda sociedade. A versão do hardware utilizado no Projeto é o Arduino R3 - ATmega328. Este conta com 14 Pinos E/S digitais podem ser usados como saídas PWM (Pulse-Width Modulation); 6 pinos entrada analógica e memória flash $32 \mathrm{~kb}$ utilizadas no projeto.

O Arduino também é responsável pelo controle do servomotor nas direções horizontais e verticais, que é uma máquina de sistema eletro-mecânico para controle de rastreamento que executa um movimento de acordo com o comando específico, verificando sua atual posição pelo sol e chegando até o local desejado, diferentemente dos servemotores de rotação contínua.

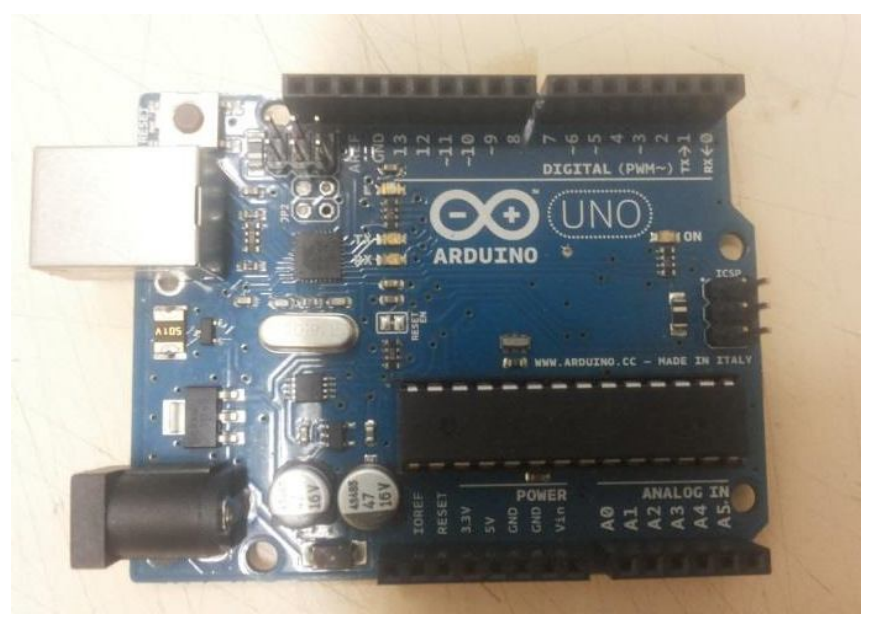

Figura 4: Placa de microcontrolador - Arduino Fonte: Autor

\subsection{Estrutura do dispositivo}

A Figura 5 ilustra o dispositivo de bancada desenvolvido, os principais componentes utilizados (LDRs, Arduino e servomotores) para proporcionar sustentação ao sistema usa-se: uma caixa de luz com 40mm em cada lado, este material foi escolhido devido sua grande facilidade de manipulação, não necessitando de soldas ou equipamentos especiais. Para este tamanho o dispositivo apresentou-se muito estável, suportando o peso do painel fotovoltaico com a vantagem de ser barato e reciclável. As dimensões finais são: comprimento de $250 \mathrm{~mm}$ em cada lado e altura de $160 \mathrm{~mm}$.

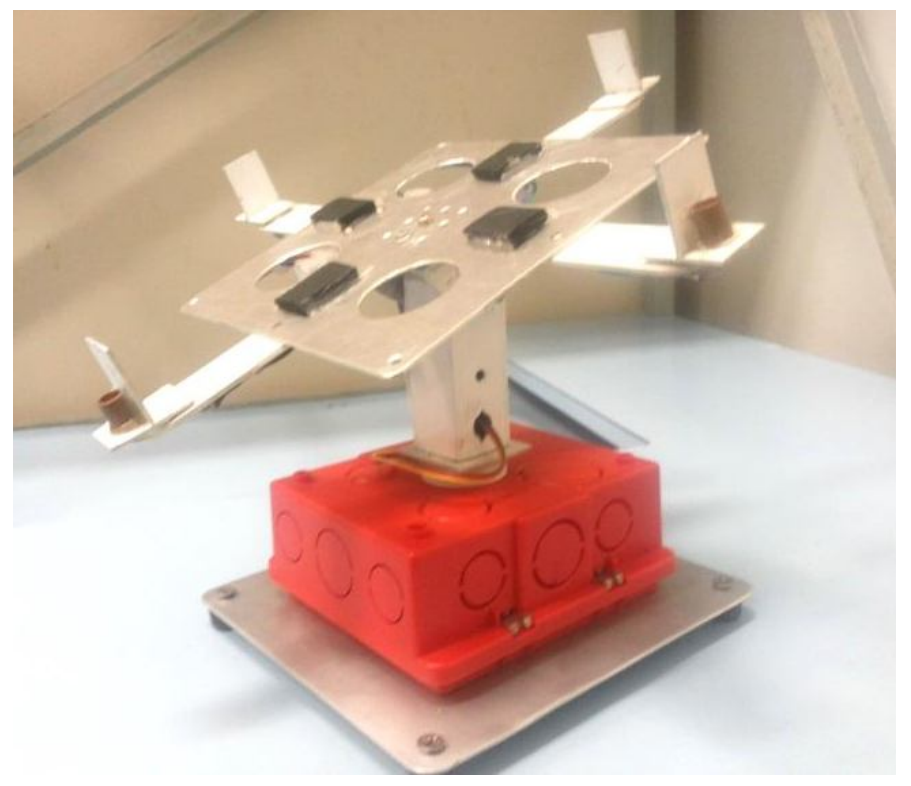

Figura 5: Versão do dispositivo Fonte: Autor 
A figura 6 apresenta as especificações do servemotor vertical e horizontal para proporcionar a redução da velocidade: $0,12 \mathrm{seg} / 60 \mathrm{Graus}$, a fim de fornecer um torque suficiente para a aplicação. É similar ao motor vertical e sua rotação de torque $4,8 \mathrm{~V}$.

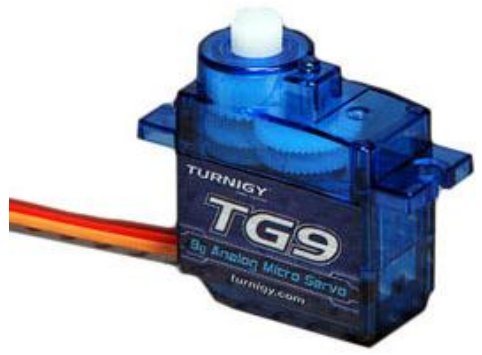

Figura 6: Micro Servo Motor 9G

\subsection{Programação em C}

A programação do microcontrolador foi feita com a utilização da linguagem de programação da plataforma de desenvolvimento do Arduino, trata-se de uma linguagem base em $\mathrm{ClC}++$. O diagrama de fluxo mostrado nos códigos 1 , 2 e 3 . Ao iniciar a execução do código, é feito uma leitura nas portas analógicas 0 e 1, e que está conectado o sensor que identifica a posição do sol. Nesta leitura são obtidos valores que podem variar entre 0 e 1023 , de acordo com a tensão sobre os LDRs.

O fluxograma 1 é a biblioteca que nos permite controlar tão facilmente os servos <Servo.h> smotor_I, que possui vários parâmetros de controle para servomotores.

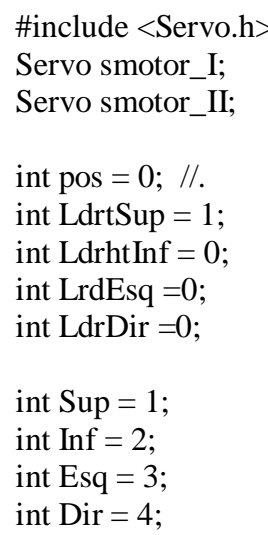

Fluxograma 1: linguagem em $\mathrm{C}$ controlar de servemotor

O código fonte permite o controle do movimento de inclinação do dispositivo com limite de considerável $90^{\circ}$, verificou se esquerda, se mover para a direta, apesar de apresentar certa instabilidade em alguns momentos podendo ser modificado para alcançar o resultado desejado.

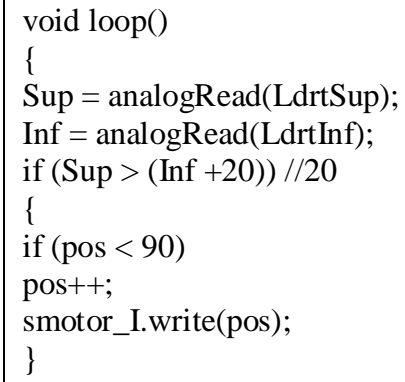

Fluxograma 2: Linguagem em C controlar de servemotor Norte/Sul 


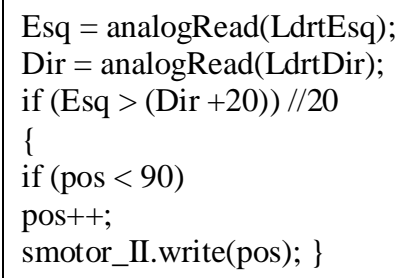

\section{Fluxograma 3: Linguagem em C controlar de servemotor Leste/Oeste}

O código fonte seria um algoritmo que ao identificar um baixo nível de o sensor da placa de sombreamento indicando o anoitecer, retomasse a posição máxima de inclinação voltando e normalmente o painel à direção do sol para o dia inteiro. $\mathrm{O}$ código fonte poderia também conter instruções de automaticamente parada move durante a noite, quando amanhecer voltando a funcionar ao visto que o código atual continua analisando o ambiente mesmo em completa ausência de luz.

\section{Resultados Conclusivos}

A presente pesquisa que utilizou um microcontrolador e materiais de baixo custo para conservar o caráter simples e versátil do equipamento conclui que produto é inovador quanto aos materiais usado para gerar o sensoriamento solar. Ainda precisa-se melhorar os parâmetros adotados e continuamente a busca por um deslocamento mais próximo ao do sol possível, através de algoritmos de rastreáveis do tipo lógica nebulosa. As principais condicionantes do projeto foram baixo custo (houve redução de em média $30 \%$ no valor quando comparado a um modelo similar vendido no mercado) e pequeno consumo de energia.

\section{AGRADECIMENTOS}

Agradecemos a Universidade Federal de Rio Grande do Norte - UFRN e ao Programa de Pós-Graduação em Engenharia Mecânica da UFRN pelo suporte fornecido a pesquisa.

\section{REFERÊNCIAS}

ENERGIA SOLAR. CRESESB e Atlas Solarimétrico do Brasil. Disponível em: <http://www.aneel.gov.br/aplicacoes/atlas/pdf/03-Energia_Solar\%283\%29.pdf> Acesso em: 29 de Jun de 2014.

PAIVA, Edinei Canuto. Desenvolvimento de um Rastreador Solar Microcontrolado para um Coletor Solar Concentrador. 24 à 49 pág. Tese apresentada à Universidade Federal de Viçosa. 2009. Disponível em: <http://www.tede.ufv.br/tedesimplificado/tde_arquivos/12/TDE-2013-04-11T131956Z4418/Publico/texto\%20completo.pdf>. Acesso em 29 de Jun de 2014.

FALCÃO, F. O.; CORNWALL, C. Instalação Sistemas Solares Fotovoltaicos. Eletrônica. Disponível em: http://www.electronica-pt.com/content/view/273/>. Acesso em: 29 de Jun de 2014.

PROENÇA, Emanuel Dâmaso Rodrigues Brinquete. A Energia Solar Fotovoltaica em Portugal Estado-da-Arte e Perspectivas de Desenvolvimento. Disponível em: <https://fenix.tecnico.ulisboa.pt/downloadFile/395137487931/Tese\%20\%20A\%20Energia\%20Solar\%20Fotovoltaica\%20em\%20Portugal.pdf> Acesso em: 30 de Jun de 2014.

Projeto Heliotropismo Mecânico. Engenharia de Computação, da Pontifícia Universidade Católica do Paraná. Disponível em: <http://engcomp.penbible.com/sites/default/files/users/user335/Documentacao_Projeto_HM.pdf>. Acesso em: 05 de Jul de 2014.

ALCÂNTARA, S. M. F. Análise do Desempenho de um Painel Fotovoltaico de Baixa Tensão Acoplado a um Conversor DC-DC: Topologia Booster. Disponível em: <http://www.abcm.org.br/pt/wpcontent/anais/conem/2010/PDF/CON10-1655.pdf>. Acesso em: 08 de Jul de 2014.

\section{RESPONSABILIDADE AUTORAL}

“Os autores são os únicos responsáveis pelo conteúdo deste trabalho”. 
Congresso Nacional de Matemática Aplicada à Indústria, 18 a 21 de novembro de 2014, Caldas Novas - GO

\title{
USE OF AUTOMATED SYSTEMS TO OPTIMIZE ENERGY CAPTURE IN SOLAR PANELS
}

\author{
Medeiros, I. P. M., isaacmedeiros.rn@gmail.com ${ }^{1}$ \\ Fontes, F. A. O., franciscofontes@uol.com.br ${ }^{1}$ \\ Lima, C. M., geracao3d@gmail.com ${ }^{1}$ \\ Barbosa, C. R. F., cleiton@dem.ufrn.br ${ }^{1}$ \\ Valcacer, S. M., samara.valcacer@gmail.com² \\ Sanabio, R. G., robson.sanabio@uece.br ${ }^{2}$ \\ Dantas, V. B., valter.fisic@hotmail.com²
}

${ }^{1}$ Universidade Federal do Rio Grande do Norte - UFRN - Programa Pós-Graduação em Engenharia Mecânica PPgEM, Caixa Postal 1524 - Campus Universitário Lagoa Nova, CEP 59078-970, Natal/RN - Brasil

${ }^{2}$ Universidade Federal do Rio Grande do Norte - UFRN - Programa Pós-Graduação em Ciência e Engenharia de Materiais - PPgCEM, Caixa Postal 1524 - Campus Universitário Lagoa Nova, CEP 59078-970, Natal/RN - Brasil

\begin{abstract}
The energy sources may be conventional or alternative. Although conventional energy is characterized by low cost, alternative energy gains visibility and among them solar, being clean, quiet and able to adapt to many different places. The big problem in the use of this energy is its low efficiency in converting electrical energy generated from the solar rays. And to make their energy efficiency improve work proposes coupled to a solar panel for guidance of the movements from the sunlight on the surface of the solar cell tracking mechanism to provide this movement was used two servo motors and a system embedded with sensors light (LDR - 5mm) directly connected to servo mechanisms. The embedded software using $C$ programming language which allows the determination of the correct position of the solar panel and the use of these mechanisms are expected to collect maximum solar radiation, thereby enhancing the efficiency of the whole apparatus using a low cost.
\end{abstract}

Keywords: Automation, Solar Power, Microcontroller, Language C 